\title{
Existence and regularity of
} time-dependent global attractors for the nonclassical reaction-diffusion equations with lower forcing term

\section{Qiaozhen $\mathrm{Ma}^{*}$, Xiaoping Wang and Ling Xu}

\section{"Correspondence:}

maqzh@nwnu.edu.cn

School of Mathematics and

Statistics, Northwest Normal

University, Lanzhou, Gansu 730070, China

\begin{abstract}
Based on the notation of time-dependent attractors, we prove the existence and the regularity of time-dependent global attractors for a class of nonclassical reaction-diffusion equations when the forcing term $g(x) \in H^{-1}(\Omega)$ and the nonlinear function satisfies the critical exponent growth, which is weaker than the conditions used in (Jing and Liu in Appl. Anal. 94(7):1439-1449, 2015).
\end{abstract}

MSC: 35B25; 37L30; 45K05

Keywords: nonclassical reaction-diffusion equations; time-dependent attractors; regularity

\section{Introduction}

Recently, Conti and Di Plinio et al. [2-4] presented the notation of time-dependent global attractors and studied the long-time behavior of the wave equations and oscillation equations in the topology space equipped with the norm related to the time, respectively. Motivated by these results we investigate the existence and regularity of time-dependent global attractors for a class of nonclassical reaction-diffusion equations

$$
\begin{cases}u_{t}-\varepsilon(t) \Delta u_{t}-\Delta u+\lambda u=f(u)+g(x), & x \in \Omega, \\ \left.u\right|_{\partial \Omega}=0, & t \in \mathbb{R}, \\ u(x, \tau)=u_{\tau}(x), & t \geq \tau .\end{cases}
$$

Here $\Omega$ is a bounded set of $\mathbb{R}^{n}(n \geq 3)$ with smooth boundary $\partial \Omega$. $\lambda>0, \tau \in \mathbb{R}$, and $\varepsilon(t)$ is a decreasing bounded function satisfying

$$
\lim _{t \rightarrow+\infty} \varepsilon(t)=0
$$

and there exists $v>0$ such that

$$
\sup _{t \in \mathbb{R}}\left[|\varepsilon(t)|+\left|\varepsilon^{\prime}(t)\right|\right] \leq v .
$$

(c) $2016 \mathrm{Ma}$ et al. This article is distributed under the terms of the Creative Commons Attribution 4.0 International License (http://creativecommons.org/licenses/by/4.0/), which permits unrestricted use, distribution, and reproduction in any medium, provided you give appropriate credit to the original author(s) and the source, provide a link to the Creative Commons license, and indicate if changes were made. 
The nonlinearity $f \in C^{1}(\mathbb{R})$ with $f(0)=0$, is assumed to satisfy the following conditions:

$$
\begin{aligned}
& \limsup _{|s| \rightarrow \infty} \frac{f(s)}{s}<\lambda_{1}, \quad \forall s \in \mathbb{R}, \\
& \left|f^{\prime}(s)\right| \leq C\left(1+|s|^{\frac{4}{n-2}}\right), \quad \forall s \in \mathbb{R},
\end{aligned}
$$

where $\lambda_{1}$ is the first eigenvalue of $-\triangle$ in $H_{0}^{1}(\Omega), C$ is a positive constant.

The nonclassical reaction-diffusion equation arises as a mathematical model to describe physical phenomena, such as non-Newtonian flows, solid mechanics, and heat conduction [5-7]. Aifantis provides a quite general approach for obtaining these equations (see $[5,8])$.

When $\varepsilon(t)$ in (1.1) is only a positive constant, the long-time behavior of solutions for (1.1) has been extensively studied by several authors in [9-19] and the references therein. For instance, some authors obtained the existence of global(pullback) attractors of solutions for both the autonomous case $[9,10,12,14,16]$ and the nonautonomous case $[15,17$, 19]. Anh and Toan [18] investigated the existence and upper semicontinuity of uniform attractor in $H^{1}\left(\mathbb{R}^{\mathbb{N}}\right)$ for this problem; besides, they also considered the case of singularly oscillating external forces on $\mathbb{R}^{\mathbb{N}}$ [20]. The existence of exponential attractors was obtained in $[11,13,15]$. In the general case of a time dependence, to the best of our knowledge, only Ding and Liu [1] proved the existence and regularity of time-dependent global attractors of (1.1) when the force term $g \in L^{2}(\Omega)\left(\Omega \subset \mathbb{R}^{3}\right)$ and the nonlinear term $f$ satisfies the following conditions:

(i) $\limsup _{|s| \rightarrow \infty} \frac{f(s)}{s}<\lambda_{1}, \quad \forall s \in \mathbb{R}$,

(ii) $\quad\left|f^{\prime \prime}(s)\right| \leq C(1+|s|), \quad \forall s \in \mathbb{R}$.

In this paper, following the general lines of the approach used in [2-4], we investigate the existence and regularity of the time-dependent attractors for the process $U(t, \tau)$ generated by (1.1) under weaker conditions than [1].

\section{Preliminaries}

Without loss of generality, denote $H=L^{2}(\Omega)$ with the inner products $\langle\cdot, \cdot\rangle$ and norms $\|\cdot\|$. For $0 \leq \sigma \leq 2$, we define the hierarchy of compactly nested Hilbert spaces

$$
H_{\sigma}=D\left(A^{\frac{\sigma}{2}}\right), \quad\langle w, v\rangle_{\sigma}=\left\langle A^{\frac{\sigma}{2}} w, A^{\frac{\sigma}{2}} v\right\rangle, \quad\|w\|_{\sigma}=\left\|A^{\frac{\sigma}{2}} w\right\| .
$$

Then, for $t \in \mathbb{R}$ and $-1 \leq \sigma \leq 1$, we introduce the time-dependent spaces

$$
\mathcal{H}_{t}^{\sigma}=H_{\sigma+1}
$$

endowed with the time-dependent norms

$$
\|u\|_{\mathcal{H}_{t}^{\sigma}}^{2}=\|u\|_{\sigma}^{2}+\varepsilon(t)\|u\|_{\sigma+1}^{2} .
$$


The symbol $\sigma$ is always omitted whenever zero. In particular, the time-dependent phase space where we settle the problem is

$$
\mathcal{H}_{t}^{-1}=H_{0}=L^{2}, \quad \mathcal{H}_{t}=H_{1}, \quad \text { with }\|u\|_{\mathcal{H}_{t}}^{2}=\|u\|^{2}+\varepsilon(t)\|u\|_{1}^{2},
$$

then we have the compact embeddings

$$
\mathcal{H}_{t}^{\sigma} \Subset \mathcal{H}_{t}, \quad-1 \leq \sigma<1,
$$

with injection constants independent of $t \in \mathbb{R}$. Note that the spaces $\mathcal{H}_{t}$ are all the same as linear spaces; besides, since $\varepsilon(t)$ is a decreasing function of $t$, for every $u \in H_{1}$ and $t \geq \tau \in \mathbb{R}$ we have

$$
\|u\|_{\mathcal{H}_{t}}^{2} \leq\|u\|_{\mathcal{H}_{\tau}}^{2} \leq \max \left\{1, \frac{\varepsilon(\tau)}{\varepsilon(t)}\right\}\|u\|_{\mathcal{H}_{t}}^{2} .
$$

Hence the norms $\|u\|_{\mathcal{H}_{t}}^{2}$ and $\|u\|_{\mathcal{H}_{\tau}}^{2}$ are equivalent for any fixed $t, \tau \in \mathbb{R}$, but the equivalent constant blows up when $t \rightarrow+\infty$.

\section{The main results}

\subsection{A priori estimates}

Under the assumptions of (1.2)-(1.5), if $g \in H^{-1}(\Omega)$, then using the standard Galerkin approximation method ([21]), we can obtain the result concerning the existence and uniqueness of solution for the problem (1.1); see, for example, $[6,9,10]$. Thus, based on the subsequent Lemma 3.2 we get the following results.

Lemma 3.1 Assume that (1.2)-(1.5) hold, for any $u_{\tau} \in \mathcal{H}_{\tau}$, there is a unique solution $u$ of (1.1) satisfying

$$
u \in \mathcal{C}\left([\tau, t], H_{1}\right) .
$$

Furthermore, let $u_{i}(\tau) \in \mathcal{H}_{\tau}$ be two initial conditions such that $\left\|u_{i}(\tau)\right\|_{\mathcal{H}_{\tau}} \leq R(i=1,2)$ and denote by $u_{i}(t)$ the corresponding solutions to the problem (1.1). Then the following estimate holds:

$$
\left\|u_{1}(t)-u_{2}(t)\right\|_{\mathcal{H}_{t}} \leq e^{K(t-\tau)}\left\|u_{1}(\tau)-u_{2}(\tau)\right\|_{\mathcal{H}_{\tau}}, \quad \forall t \geq \tau
$$

for some constant $K=K(R)>0$.

Proof We only need to prove the estimate (3.1). Let $C$ be a generic positive constant depending on $R$ but independent of $u_{i}(\tau)$. We first observe that the energy estimate in Lemma 3.2 below ensures

$$
\left\|U(t, \tau) u_{i}(\tau)\right\|_{\mathcal{H}_{t}} \leq C, \quad i=1,2 .
$$

We write $u_{i}(t)=U(t, \tau) u_{i}(\tau), \bar{u}=U(t, \tau) u_{1}(\tau)-U(t, \tau) u_{2}(\tau)$. Then the difference between the two solutions satisfies

$$
\bar{u}_{t}-\varepsilon(t) \Delta \bar{u}_{t}-\Delta \bar{u}+\lambda \bar{u}=f\left(u_{1}\right)-f\left(u_{2}\right),
$$


with initial datum $u(\tau)=u_{1}(\tau)-u_{2}(\tau)$. Multiplying by $2 \bar{u}$ in $L^{2}(\Omega)$ we obtain

$$
\frac{d}{d t}\|\bar{u}\|_{\mathcal{H}_{t}}^{2}+2 \lambda\|\bar{u}\|+\left(2-\varepsilon^{\prime}(t)\right)\|\bar{u}\|_{1}^{2}=2\left\langle f\left(u_{1}\right)-f\left(u_{2}\right), \bar{u}\right\rangle .
$$

Combining with the embedding $H_{0}^{1}(\Omega) \hookrightarrow L^{2 n /(n-2)}(n \geq 3)$, and according to (1.5) and (3.2), we have

$$
\begin{aligned}
& 2 \mid\left\langle f\left(u_{1}\right)-f\left(u_{1}\right), \bar{u}||\right. \\
& \quad=2 \int_{\Omega}\left|\left(f\left(u_{1}\right)-f\left(u_{2}\right)\right) \bar{u}\right| d x=2 \int_{\Omega}\left|\left(f^{\prime}\left(\theta u_{1}+(1-\theta) u_{2}\right) \bar{u}\right) \bar{u}\right| d x \\
& \quad \leq C \int_{\Omega}\left(1+|u|^{\frac{4}{n-2}}\right)|\bar{u}|^{2} d x \leq C\left[\int_{\Omega}\left(1+|u|^{\frac{4}{n-2}}\right)^{\frac{n}{2}}\right]^{\frac{2}{n}} \cdot\left[\int_{\Omega}|\bar{u}|^{2 \cdot \frac{n}{n-2}}\right]^{\frac{n-2}{n}} \\
& \quad \leq C\left(1+\|u\|_{L^{2 n(n-2)}}^{4 /(n-2)}\right)\|\bar{u}\|_{L^{2 n /(n-2)}}^{2} \leq C\|\bar{u}\|_{1}^{2} .
\end{aligned}
$$

Thus, we end up with the differential inequality

$$
\frac{d}{d t}\|\bar{u}\|_{\mathcal{H}_{t}}^{2} \leq C\|\bar{u}\|_{\mathcal{H}_{t}}^{2}
$$

and an application of the Gronwall lemma on $[\tau, t]$ completes the proof.

By means of the Lemma 3.1, a family of maps with $t \geq \tau \in \mathbb{R}$

$$
U(t, \tau): H_{\tau} \rightarrow H_{t} \quad \text { acting as } U(t, \tau) u_{\tau}=u(t)
$$

define a strongly continuous process on a family of spaces $\left\{\mathcal{H}_{t}\right\}_{t \in \mathbb{R}}$.

Lemma 3.2 Assume that (1.2)-(1.5) hold. For any $u_{\tau} \in \mathcal{H}_{\tau}, t \geq \tau$, let $U(t, \tau) u_{\tau}$ be the solution of (1.1) with initial value $u_{\tau}$. Then there is a positive constant $K$, such that

$$
\left\|U(t, \tau) u_{\tau}\right\|_{\mathcal{H}_{t}} \leq K, \quad \forall t \geq \tau
$$

Proof Multiplying (1.1) by $2 u+2 u_{t}$ in $H$ we obtain

$$
\begin{aligned}
& \frac{d}{d t}\left[(1+\lambda)\|u\|^{2}+(1+\varepsilon(t))\|u\|_{1}^{2}-2\langle F(u), 1\rangle-2\langle g, u\rangle\right] \\
& \quad+2 \lambda\|u\|^{2}+\left(2-\varepsilon^{\prime}(t)\right)\|u\|_{1}^{2}-2\langle f(u), u\rangle-2\langle g, u\rangle+2\left\|u_{t}\right\|^{2}+2 \varepsilon(t)\left\|u_{t}\right\|_{1}^{2}=0 .
\end{aligned}
$$

Let

$$
\begin{aligned}
& E(t)=(1+\lambda)\|u\|^{2}+(1+\varepsilon(t))\|u\|_{1}^{2}-2\langle F(u), 1\rangle-2\langle g, u\rangle, \\
& I(t)=2 \lambda\|u\|^{2}+\left(2-\varepsilon^{\prime}(t)\right)\|u\|_{1}^{2}-2\langle f(u), u\rangle-2\langle g, u\rangle,
\end{aligned}
$$

it yields

$$
\frac{d}{d t} E(t)+I(t) \leq 0
$$


namely

$$
E(t) \leq-\int_{\tau}^{t} I(s) d s+E(\tau)
$$

where

$$
E(\tau)=(1+\lambda)\left\|u_{\tau}\right\|^{2}+(1+\varepsilon(\tau))\left\|u_{\tau}\right\|_{1}^{2}-2\left\langle F\left(u_{\tau}\right), 1\right\rangle-2\left\langle g, u_{\tau}\right\rangle .
$$

In view of the condition (1.5), there are $0<v<1$ and $c \geq 0$, such that

$$
\begin{aligned}
& 2\langle F(u), 1\rangle \leq(1-v)\|u\|_{1}^{2}+c, \\
& \langle f(u), u\rangle \leq(1-v)\|u\|_{1}^{2}+c, \quad \forall u \in H_{1} .
\end{aligned}
$$

Thus, combining with (1.4), (3.4), and (3.5), there exist two positive constants $M_{1}$ and $M_{2}$, such that

$$
\begin{aligned}
E(t) & \geq(1+\lambda)\|u\|^{2}+(1+\varepsilon(t))\|u\|_{1}^{2}-(1-v)\|u\|_{1}^{2}-c-\frac{2}{v}\|g\|_{H^{-1}}^{2}-\frac{v}{2}\|u\|_{1}^{2} \\
& \geq \lambda\|u\|^{2}+\left(\frac{v}{2}+\varepsilon(t)\right)\|u\|_{1}^{2}-M_{1}
\end{aligned}
$$

and

$$
\begin{aligned}
I(t) & \geq 2 \lambda\|u\|^{2}+\left(2-\varepsilon^{\prime}(t)\right)\|u\|_{1}^{2}-2(1-v)\|u\|_{1}^{2}-2 c-\frac{2}{v}\|g\|_{H^{-1}}^{2}-\frac{v}{2}\|u\|_{1}^{2} \\
& \geq \lambda\|u\|^{2}+\left(\frac{v}{2}+\varepsilon(t)\right)\|u\|_{1}^{2}-M_{2} .
\end{aligned}
$$

So we deduce that

$$
\begin{aligned}
& \lambda\|u(t)\|^{2}+\left(\frac{v}{2}+\varepsilon(t)\right)\|u(t)\|_{1}^{2}-M_{1} \\
& \quad \leq-\int_{\tau}^{t}\left(\lambda\|u(s)\|^{2}+\left(\frac{v}{2}+\varepsilon(s)\right)\|u(s)\|_{1}^{2}-M_{2}\right) d s+E(\tau) .
\end{aligned}
$$

Therefore, for any $K>M_{2}$, there exists $t_{0}>\tau$ such that

$$
\lambda\left\|u\left(t_{0}\right)\right\|^{2}+\left(\frac{v}{2}+\varepsilon\left(t_{0}\right)\right)\left\|u\left(t_{0}\right)\right\|_{1}^{2} \leq K .
$$

As a result, if $u$ is a solution of the systems (1.1), if we let $B_{t}=\bigcup_{t \geq \tau} U(t, \tau) B_{\tau}$, where

$$
B_{\tau}=\left\{u_{\tau} \in \mathbb{B}_{\tau}(R): \lambda\left\|u_{\tau}\right\|^{2}+\left(\frac{v}{2}+\varepsilon(\tau)\right)\left\|u_{\tau}\right\|_{1}^{2} \leq K\right\},
$$

then $B_{t}$ is a bounded time-dependent absorbing set of $\{U(t, \tau)\}_{t \geq \tau}$. Moreover, $B_{t}$ is positively invariant. 
On the other hand, from the above discussion, for every $R \geq 0$ there exist positive constants $\mu$ and $t_{0}=t_{0}(R)$ such that

$$
\lambda\|u(t)\|^{2}+(1+\varepsilon(t))\|u(t)\|_{1}^{2} \leq \mu^{2}, \quad \forall \tau \leq t-t_{0} .
$$

\subsection{The time-dependent global attractors and regularity}

The main result concerning the asymptotic behavior of problem (1.1) is contained in the following theorem.

Theorem 3.3 The process $U(t, \tau)$ generated by problem (1.1) admits an invariant timedependent global attractor $\mathcal{U}=\left\{A_{t}\right\}_{t \in \mathbb{R}}$ in $\mathcal{H}_{t}$. Besides, $A_{t}$ is bounded in $\mathcal{H}_{t}^{1}$, with a bound independent of $t$.

In order to show that the process is asymptotically compact, we shall exhibit a pullback attracting family of (non-void) compact sets. For this purpose, we exploit a suitable decomposition of the process in the sum of a decaying part and of a compact one.

\subsubsection{The decomposition}

Under the conditions (1.4)-(1.5), like in [15] we write $f=f_{0}+f_{1}$, where $f_{0}, f_{1} \in C^{1}(\mathbb{R})$ fulfill, respectively, for some $k \geq 0$,

$$
\begin{aligned}
& \left|f_{0}(s)\right| \leq C\left(1+|s|^{\frac{n+2}{n-2}}\right), \quad \forall s \in \mathbb{R}, \\
& f_{0}(s) s \leq 0, \quad \forall s \in \mathbb{R}, \\
& \left|f_{1}(s)\right| \leq C\left(1+|s|^{\gamma}\right), \quad \forall s \in \mathbb{R}, 0<\gamma<\frac{n+2}{n-2}, \\
& \limsup _{|s| \rightarrow \infty} \frac{f_{1}(s)}{s}<\lambda_{1}, \quad \forall s \in \mathbb{R} .
\end{aligned}
$$

Since the injection $i: L^{2}(\Omega) \hookrightarrow H^{-1}(\Omega)$ is dense, we know that for every $g \in H^{-1}(\Omega)$ and any $\eta>0$, there is a $g^{\eta} \in L^{2}(\Omega)$ which depends on $g$ and $\eta$ such that

$$
\left\|g-g^{\eta}\right\|_{H^{-1}}<\eta
$$

Let $\mathfrak{B}=\left\{\mathbb{B}_{t}\left(R_{0}\right)\right\}_{t \in \mathbb{R}}$ be a time-dependent absorbing set as in Lemma 3.2 and let $\tau \in \mathbb{R}$ be fixed. Then, for any $u_{\tau} \in \mathbb{B}_{\tau}\left(R_{0}\right)$, we divide $U(t, \tau) u_{\tau}$ into the sum

$$
U(t, \tau) u_{\tau}=u(t)=U_{0}(t, \tau) u_{\tau}+U_{1}(t, \tau) u_{\tau}
$$

where

$$
U_{0}(t, \tau) u_{\tau}=v^{\eta}(t), \quad U_{1}(t, \tau) u_{\tau}=w^{\eta}(t)
$$

respectively, solve the following systems:

$$
\left\{\begin{array}{l}
v_{t}^{\eta}+\varepsilon(t) A v_{t}^{\eta}+A v^{\eta}+\lambda v^{\eta}=f_{0}\left(v^{\eta}\right)+g-g^{\eta}, \quad x \in \Omega, \\
U_{0}(\tau, \tau)=u_{\tau}
\end{array}\right.
$$


and

$$
\left\{\begin{array}{l}
w_{t}^{\eta}+\varepsilon(t) A w_{t}^{\eta}+A w^{\eta}+\lambda w^{\eta}=f(u)-f_{0}\left(v^{\eta}\right)+g^{\eta}, \quad x \in \Omega \\
U_{1}(\tau, \tau)=0
\end{array}\right.
$$

In the following, the generic constant $C \geq 0$ depends only on $\mathfrak{B}$.

Lemma 3.4 Under the conditions (1.2)-(1.5), there exist two constants $\delta>0$ and $K_{1}>0$ such that

$$
\left\|U_{0}(t, \tau) u_{\tau}\right\|_{\mathcal{H}_{t}} \leq C e^{-\delta(t-\tau)}+K_{1}, \quad \forall t \geq \tau
$$

Proof Multiplying (3.12) by $2 v^{\eta}$ in $H$ we obtain

$$
\frac{d}{d t}\left[\left\|v^{\eta}\right\|^{2}+\varepsilon(t)\left\|v^{\eta}\right\|_{1}^{2}\right]+2 \lambda\left\|v^{\eta}\right\|^{2}+\left(2-\varepsilon^{\prime}(t)\right)\left\|v^{\eta}\right\|_{1}^{2}=2\left\langle f_{0}\left(v^{\eta}\right)+g-g^{\eta}, v^{\eta}\right\rangle .
$$

By (3.5), we have

$$
2\left\langle f_{0}\left(v^{\eta}\right), v^{\eta}\right\rangle=2 \int_{\Omega} f_{0}\left(v^{\eta}\right) v^{\eta} d x \leq 0,
$$

and using the Cauchy and Young inequalities we get

$$
2\left\langle g-g^{\eta}, v^{\eta}\right\rangle \leq 2\left\|g-g^{\eta}\right\|_{H^{-1}}\left\|v^{\eta}\right\|_{H^{1}} \leq\left\|g-g^{\eta}\right\|_{H^{-1}}^{2}+\left\|v^{\eta}\right\|_{1}^{2} .
$$

In view of (1.3) we get $1-\varepsilon^{\prime}(t) \geq \varepsilon(t)>0$, thus we find

$$
\frac{d}{d t}\left(\varepsilon(t)\left\|v^{\eta}\right\|^{2}+\left\|v^{\eta}\right\|_{1}^{2}\right)+2 \lambda\left\|v^{\eta}\right\|^{2}+\varepsilon(t)\left\|v^{\eta}\right\|_{1}^{2} \leq \eta^{2}
$$

Taking $\delta=\min \{2 \lambda, 1\}>0$, then

$$
\frac{d}{d t}\left\|U_{0}(t, \tau) u_{\tau}\right\|_{\mathcal{H}_{t}}^{2}+\delta\left\|U_{0}(t, \tau) u_{\tau}\right\|_{\mathcal{H}_{t}}^{2} \leq \eta^{2}
$$

Applying the Gronwall lemma on the interval $[\tau, t]$ with $t \geq \tau$, it follows that

$$
\left\|U_{0}(t, \tau) u_{\tau}\right\|_{\mathcal{H}_{t}}^{2} \leq\left\|u_{\tau}\right\|_{\mathcal{H}_{\tau}}^{2} e^{-\delta(t-\tau)}+\eta^{2} / \delta
$$

The proof is complete.

Summing up, the following uniform boundedness holds:

$$
\sup _{t \geq \tau}\left[\left\|U(t, \tau) u_{\tau}\right\|_{\mathcal{H}_{t}}+\left\|U_{0}(t, \tau) u_{\tau}\right\|_{\mathcal{H}_{t}}+\left\|U_{1}(t, \tau) u_{\tau}\right\|_{\mathcal{H}_{t}}\right] \leq C
$$

In order to prove our further result, we also need the condition

$$
\limsup _{|s| \rightarrow \infty} f^{\prime}(s)<\lambda_{1}, \quad \forall s \in \mathbb{R} .
$$


Lemma 3.5 Under the conditions (1.2)-(1.4) and (3.15), there exists $M=M(\mathfrak{B})>0$ such that

$$
\sup _{t \geq \tau}\left\|U_{1}(t, \tau) u_{\tau}\right\|_{\mathcal{H}_{t}^{1 / 3}} \leq M
$$

Proof Multiplying (3.13) by $2 A^{1 / 3} w^{\eta}$ in $H$ we have

$$
\begin{aligned}
\frac{d}{d t} & {\left[\left\|w^{\eta}\right\|_{1 / 3}^{2}+\varepsilon(t)\left\|w^{\eta}\right\|_{4 / 3}^{2}\right]+2 \lambda\left\|w^{\eta}\right\|_{1 / 3}^{2}+\left(2-\varepsilon^{\prime}(t)\right)\left\|w^{\eta}\right\|_{4 / 3}^{2} } \\
& =2\left\langle f(u)-f_{0}\left(v^{\eta}\right)+g^{\eta}, A^{1 / 3} w^{\eta}\right\rangle \\
& =2\left\langle f(u)-f\left(v^{\eta}\right), A^{1 / 3} w^{\eta}\right\rangle+2\left\langle f_{1}\left(v^{\eta}\right), A^{1 / 3} w^{\eta}\right\rangle+2\left\langle g^{\eta}, A^{1 / 3} w^{\eta}\right\rangle .
\end{aligned}
$$

Using the Young equality, it leads to

$$
\begin{aligned}
2\left(f(u)-f\left(v^{\eta}\right), A^{1 / 3} w^{\eta}\right\rangle & =2 \int_{\Omega}\left(f(u)-f\left(v^{\eta}\right)\right) A^{1 / 3} w^{\eta} d x \\
& \leq 2 \int_{\Omega}\left|f^{\prime}\left(u-\theta\left(u-v^{\eta}\right)\right)\left\|u-v^{\eta}\right\| A^{1 / 3} w^{\eta}\right| d x \\
& \leq C \int_{\Omega}\left|w^{\eta}\left\|A^{1 / 3} w^{\eta} \mid d x \leq C\right\| w^{\eta}\|\| A^{1 / 3} w^{\eta}\left\|\leq C+\frac{1}{4}\right\| w^{\eta} \|_{4 / 3}^{2} .\right.
\end{aligned}
$$

Since $\frac{3(n-2) \gamma}{3 n+4}<1$, from (3.9) it follows that

$$
\begin{aligned}
& 2\left|\left\langle f_{1}\left(v^{\eta}\right), A^{1 / 3} w^{\eta}\right\rangle\right| \\
& \quad \leq C \int_{\Omega}\left(1+\left|v^{\eta}\right|^{\gamma}\right)\left|A^{1 / 3} w^{\eta}\right| d x \leq C\left(\int_{\Omega}\left(1+\left|v^{\eta}\right|^{\gamma}\right)^{\frac{6 n}{3 n+4}}\right)^{\frac{3 n+4}{6 n}}\left(\int_{\Omega}\left|A^{1 / 3} w^{\eta}\right|^{\frac{6 n}{3 n-4}}\right)^{\frac{3 n-4}{6 n}} \\
& \quad \leq C\left(1+\left\|v^{\eta}\right\|_{L^{6 n \gamma /(3 n+4)}}^{\gamma}\right)\left\|A^{1 / 3} w^{\eta}\right\|_{L^{6 n /(3 n-4)}} \leq C\left(1+\left\|v^{\eta}\right\|_{1}^{\gamma}\right)\left\|A^{2 / 3} w^{\eta}\right\| \\
& \quad \leq C\left\|w^{\eta}\right\|_{4 / 3} \leq C+\frac{1}{4}\left\|w^{\eta}\right\|_{4 / 3}^{2}
\end{aligned}
$$

where we have used the embedding $H_{1}=D\left(A^{\frac{1}{2}}\right) \hookrightarrow L^{\frac{2 n}{n-2}}$ with the facts that $\frac{6 n \gamma}{3 n+4} \leq \frac{2 n}{n-2}$ and $H_{2 / 3}=D\left(A^{1 / 3}\right) \hookrightarrow L^{\frac{6 n}{3 n-4}}$. Moreover, making use of the embedding $H_{2 / 3} \subset L^{18 / 5}(\Omega)$, we have

$$
2\left\langle g^{\eta}, A^{1 / 3} w^{\eta}\right\rangle \leq 2\left\|g^{\eta}\right\|_{L^{18 / 13}}\left\|A^{1 / 3} w^{\eta}\right\|_{L^{18 / 5}} \leq C\left\|g^{\eta}\right\|\left\|w^{\eta}\right\|_{4 / 3} \leq C\left\|g^{\eta}\right\|^{2}+\frac{1}{2}\left\|w^{\eta}\right\|_{4 / 3}^{2} .
$$

As a result, we deduce

$$
\frac{d}{d t}\left(\left\|w^{\eta}\right\|_{1 / 3}^{2}+\varepsilon(t)\left\|w^{\eta}\right\|_{4 / 3}^{2}\right)+2 \lambda\left\|w^{\eta}\right\|_{1 / 3}^{2}+\left(1-\varepsilon^{\prime}(t)\right)\left\|w^{\eta}\right\|_{4 / 3}^{2} \leq C .
$$

Using $1-\varepsilon^{\prime}(t) \geq \varepsilon(t)>0$, we conclude

$$
\frac{d}{d t}\left(\left\|w^{\eta}\right\|_{1 / 3}^{2}+\varepsilon(t)\left\|w^{\eta}\right\|_{4 / 3}^{2}\right)+2 \lambda\left\|w^{\eta}\right\|_{1 / 3}^{2}+\varepsilon(t)\left\|w^{\eta}\right\|_{4 / 3}^{2} \leq C .
$$

Taking $\delta=\min \{2 \lambda, 1\}>0$, we have

$$
\frac{d}{d t}\left\|U_{1}(t, \tau) u_{\tau}\right\|_{\mathcal{H}_{t}}^{2}+\delta\left\|U_{1}(t, \tau) u_{\tau}\right\|_{\mathcal{H}_{t}}^{2} \leq C
$$


Applying the Gronwall lemma on the interval $[\tau, t]$ with $t \geq \tau$ we obtain

$$
\left\|U_{1}(t, \tau) u_{\tau}\right\|_{\mathcal{H}_{t}^{1 / 3}}^{2} \leq\left\|u_{\tau}\right\|_{\mathcal{H}_{\tau}^{1 / 3}}^{2} e^{-\delta(t-\tau)}+C / \delta
$$

The proof is complete.

\subsubsection{Existence of the invariant attractor}

In line with the Lemma 3.5, we consider a family of $\mathcal{K}=\left\{K_{t}\right\}_{t \in \mathbb{R}}$, where

$$
K_{t}=\left\{u(t) \in \mathcal{H}_{t}^{1 / 3}:\|u(t)\|_{\mathcal{H}_{t}^{1 / 3}} \leq M\right\} .
$$

It is clear that $K_{t}$ is compact since embedding $\mathcal{H}_{t}^{1 / 3} \Subset \mathcal{H}_{t}$ is compact; besides, since the injection constants are independent of $t, \mathcal{K}$ is uniform. Finally, Lemma 3.2, Lemma 3.4, and Lemma 3.5 imply that $\mathcal{K}$ is pullback attracting; indeed,

$$
\delta_{t}\left(\mathbb{B}_{\tau}\left(R_{0}\right), K_{t}\right) \leq C e^{-\delta(t-\tau)}, \quad \forall t \geq \tau,
$$

where $\delta_{t}(B, C)$ is the Hausdorff semidistance of two nonempty sets $B, C$.

Hence the process $U(t, \tau)$ is asymptotically compact, which proves the existence of the unique time-dependent global attractor $U=\left\{A_{t}\right\}_{t \in \mathbb{R}}$. The invariance of $U$ follows by the strong continuity of the process stated in Lemma 3.1 .

\subsubsection{Regularity of the attractor}

The minimality of $\mathcal{U}$ in $\mathcal{K}$ establishes that $A_{t} \subset K_{t}$ for all $t \in \mathbb{R}$. Therefore, we immediately obtain the following regularity result.

Lemma 3.6 $A_{t}$ is bounded in $\mathcal{H}_{t}^{1 / 3}$ (with a bound independent of $t$ ).

To prove that $A_{t}$ is uniformly bounded in $\mathcal{H}_{t}^{1}$, as claimed in Theorem 3.3, we argue as follows. Fix $\tau \in \mathbb{R}$, for $u_{\tau} \in A_{\tau}$, we split the solution $U(t, \tau) u_{\tau}=u(t)$ into the sum $U_{0}(t, \tau) u_{\tau}+U_{1}(t, \tau) u_{\tau}$, where $U_{0}(t, \tau) u_{\tau}=v^{\eta}(t)$ and $U_{1}(t, \tau) u_{\tau}=w^{\eta}(t)$, instead of (3.12)(3.13), solving, respectively,

$$
\begin{aligned}
& \left\{\begin{array}{l}
v_{t}^{\eta}+\varepsilon(t) A v_{t}^{\eta}+A v^{\eta}+\lambda v^{\eta}=g-g^{\eta}, \quad x \in \Omega, \\
U_{0}(\tau, \tau)=u_{\tau},
\end{array}\right. \\
& \left\{\begin{array}{l}
w_{t}^{\eta}+\varepsilon(t) A w_{t}^{\eta}+A w^{\eta}+\lambda w^{\eta}=f(u)+g^{\eta}, \quad x \in \Omega, \\
U_{1}(\tau, \tau)=0 .
\end{array}\right.
\end{aligned}
$$

As a particular case of Lemma 3.4, we know that

$$
\left\|U_{0}(t, \tau) u_{\tau}\right\|_{\mathcal{H}_{t}^{1}} \leq C e^{-\delta(t-\tau)}+\eta^{2} / \delta, \quad \forall t \geq \tau .
$$

Lemma 3.7 Under the assumptions (1.2)-(1.5), the following estimate holds:

$$
\sup _{t \geq \tau}\left\|U_{1}(t, \tau) u_{\tau}\right\|_{\mathcal{H}_{t}^{1}} \leq M_{1}
$$

for some $M_{1}=M_{1}(U)>0$. 
Proof Multiplying (3.17) by $2 A w^{\eta}$ in $H$ we obtain

$$
\frac{d}{d t}\left[\left\|w^{\eta}\right\|_{1}^{2}+\varepsilon(t)\left\|w^{\eta}\right\|_{2}^{2}\right]+2 \lambda\left\|w^{\eta}\right\|_{1}^{2}+\left(2-\varepsilon^{\prime}(t)\right)\left\|w^{\eta}\right\|_{2}^{2}=2\left\langle f(u)+g^{\eta}, A w^{\eta}\right\rangle
$$

Together with embeddings $H_{1}(\Omega) \hookrightarrow L^{6}(\Omega)$, Lemma 3.2, and (3.15), we have

$$
\begin{gathered}
2\left|\left\langle f(u), A w^{\eta}\right\rangle\right|=2 \int_{\Omega}\left|f^{\prime}(u) \nabla u \cdot A^{1 / 2} w^{\eta}\right| d x \leq 2 \lambda_{1} \int_{\Omega}\left|\nabla u \cdot A^{1 / 2} w^{\eta}\right| d x \\
\leq 2 \lambda_{1}\|\nabla u\|\left\|A^{1 / 2} w^{\eta}\right\| \leq C+\frac{1}{2}\left\|w^{\eta}\right\|_{2}^{2} \\
2\left\langle g^{\eta}, A w^{\eta}\right\rangle \leq 2\left\|g^{\eta}\right\|\left\|A w^{\eta}\right\| \leq 4\left\|g^{\eta}\right\|^{2}+\frac{1}{2}\left\|w^{\eta}\right\|_{2}^{2}
\end{gathered}
$$

Therefore, we conclude

$$
\frac{d}{d t}\left(\left\|w^{\eta}\right\|_{1}^{2}+\varepsilon(t)\left\|w^{\eta}\right\|_{2}^{2}\right)+2 \lambda\left\|w^{\eta}\right\|_{1}^{2}+\left(1-\varepsilon^{\prime}(t)\right)\left\|w^{\eta}\right\|_{2}^{2} \leq C
$$

From (1.3) we have $1-\varepsilon^{\prime}(t) \geq \varepsilon(t)>0$, so we deduce

$$
\frac{d}{d t}\left\|U_{1}(t, \tau) u_{\tau}\right\|_{\mathcal{H}_{t}^{1}}^{2}+\delta\left\|U_{1}(t, \tau) u_{\tau}\right\|_{\mathcal{H}_{t}^{1}}^{2} \leq C
$$

Applying the Gronwall lemma on the interval $[\tau, t]$ with $t \geq \tau$, we obtain

$$
\left\|U_{1}(t, \tau) u_{\tau}\right\|_{\mathcal{H}_{t}^{1}}^{2} \leq\left\|u_{\tau}\right\|_{\mathcal{H}_{\tau}^{1}}^{2} e^{-\delta(t-\tau)}+C / \delta
$$

The proof is complete.

Proof of Theorem 3.3 For all $t \in \mathbb{R}$, inequality (3.18) and Lemma 3.7 imply that

$$
\lim _{\tau \rightarrow-\infty} \delta_{t}\left(U(t, \tau) A_{\tau}, K_{t}^{1}\right)=0
$$

where

$$
K_{t}^{1}=\left\{u(t) \in \mathcal{H}_{t}^{1}:\|u(t)\|_{\mathcal{H}_{t}^{1}} \leq M_{1}\right\}
$$

Since $U$ is invariant, this means

$$
\delta_{t}\left(A_{t}, K_{t}^{1}\right)=0
$$

Hence, $A_{t} \subset \overline{K_{t}^{1}}=K_{t}^{1}$; that is, $A_{t}$ is bounded in $\mathcal{H}_{t}^{1}$ with a bound independent of $t \in \mathbb{R}$. 


\section{Acknowledgements}

This work was partly supported by the NSFC $(11561064,11361053)$ and the NSF of Gansu Province (145RJZA112), in part by the Fundamental Research Funds of Gansu Universities.

Received: 21 October 2015 Accepted: 30 December 2015 Published online: 13 January 2016

\section{References}

1. Jing, D, Liu, YF: Time-dependent global attractor for the nonclassical diffusion equations. Appl. Anal. 94(7), 1439-1449 (2015)

2. Di Plinio, F, Duane, GS, Temam, R: Time-dependent attractor for the oscillation equation. Discrete Contin. Dyn. Syst. 29, 141-167 (2011)

3. Conti, M, Pata, V, Temam, R: Attractors for processes on time-dependent spaces. Applications to wave equation J. Differ. Equ. 255, 1254-1277 (2013)

4. Conti, M, Pata, V: Asymptotic structure of the attractor for processes on time-dependent spaces. Nonlinear Anal., Real World Appl. 19, 1-10 (2014)

5. Aifantis, EC: On the problem of diffusion in solids. Acta Mech. 37, 265-296 (1980)

6. Kuttler, K, Aifantis, E: Quasilinear evolution equation in nonclassical diffusion. SIAM J. Math. Anal. 19, 110-120 (1988)

7. Chen, PJ, Gurtin, ME: On a theory of heat conduction involving two temperatures. Z. Angew. Math. Phys. 19, 614-627 (1968)

8. Aifantis, EC: Gradient nanomechanics: applications to deformation, fracture, and diffusion in nanopolycrystals. Metall. Trans. A, Phys. Metall. Mater. Sci. 42(10), 2985-2998 (2011)

9. Xiao, YL: Attractors for a nonclassical diffusion equation. Acta Math. Appl. Sinica (Engl. Ser.) 18, 273-276 (2002)

10. Sun, CY, Wang, SY, Zhong, CK: Global attractors for a nonclassical diffusion equation. Acta Math. Sin. Engl. Ser. 23, 1271-1280 (2007)

11. Liu, YF, Ma, QZ: Exponential attractors for a nonclassical diffusion equation. Electron. J. Differ. Equ. 2009, 9 (2009)

12. $\mathrm{Ma}, \mathrm{QZ}, \mathrm{Liu}, \mathrm{YF}, \mathrm{Zhang}, \mathrm{FH}$ : Global attractors in $H_{1}\left(\mathbb{R}^{N}\right)$ for nonclassical diffusion equation. Discrete Dyn. Nat. Soc 2012, Article ID 672762 (2012)

13. Zhang, YJ, Ma, QZ: Exponential attractors for nonclassical diffusion equation with lower regular forcing term. Int. J. Mod. Nonlinear Theory Appl. 3, 15-22 (2014)

14. Wang, SY, Li, DS, Zhong, CK: On the dynamics of a class of nonclassical parabolic equations. J. Math. Anal. Appl. 317, 565-582 (2006)

15. Sun, CY, Yang, MH: Dynamics of the nonclassical diffusion equations. Asymptot. Anal. 59, 51-81 (2008)

16. $\mathrm{Wu}, \mathrm{HQ}$, Zhang, ZY: Asymptotic regularity for the nonclassical diffusion equation with lower regular forcing term. Dyn. Syst. 26, 391-400 (2011)

17. Zhang, FH, Liu, YF: Pullback attractors in $H^{1}\left(R^{N}\right)$ for non-autonomous nonclassical diffusion equations. Dyn. Syst. 29, 106-118 (2014)

18. Anh, CT, Toan, ND: Existence and upper semicontinuity of uniform attractors in $H^{1}\left(\mathbb{R}^{N}\right)$ for nonautonomous nonclassical diffusion equations. Ann. Pol. Math. 111(3), 271-295 (2014)

19. Anh, CT, Bao, TQ: Dynamics of non-autonomous nonclassical diffusion equations on $\mathbb{R}^{N}$. Commun. Pure Appl. Anal. $11,1231-1252(2012)$

20. Anh, $C T$, Tona, ND: Nonclassical diffusion equations on $\mathbb{R}^{N}$ with singularly oscillating external forces. Appl. Math. Lett. 38, 20-26 (2014)

21. Temam, R: Infinite-Dimensional Dynamical Systems in Mechanics and Physic. Springer, New York (1997)

\section{Submit your manuscript to a SpringerOpen ${ }^{\circ}$ journal and benefit from:}

- Convenient online submission

Rigorous peer review

- Immediate publication on acceptance

- Open access: articles freely available online

- High visibility within the field

- Retaining the copyright to your article 